\title{
Novel Chitosan Microparticles for Sequestration of Overdosed Drug
}

\author{
Soma Chakraborty*, Patricia Teresa F. Agbayani, Modesto T. Chua \\ Department of Chemistry, School of Science and Engineering, Loyola Schools, Ateneo de Manila University, \\ Loyola Heights, Quezon City 1108
}

\begin{abstract}
Chitosan microparticles were synthesized by crosslinking chitosan and glycidyl trimethylammonium chloride modified chitosan inside the reverse micelles of Span and Tween 80. The particles were of uniform size with the average diameter of $10 \mu \mathrm{m}$. The particles swelled almost twice the original size in $20 \mathrm{~min}$ when dispersed in a buffer solution of $\mathrm{pH}$ 7.4. The feasibility of chitosan microparticles as drug sequestrant was tested using propafenone as the model drug. The particles sequestered free propafenone from a buffer solution of pH 7.4. In 20 min unmodified chitosan and modified chitosan microparticles sequestered 53\% and $51.5 \%$ of the free drug respectively. The amount of drug sequestered increased with increase in the initial free drug concentration. The presence of normal saline also improved propafenone sequestration.
\end{abstract}

Keywords: chitosan; reverse micelles; microparticles; drug sequestration

\section{INTRODUCTION}

Drug intoxication, developed as a result of accidental overdosing or of suicide attempts, is a serious health problem. Hence novel methods to remedy drug overdose are continuously being developed in order to make treatment simpler and more effective. The common methods used to counter drug overdosing are via mechanical pumping of the stomach, oral administration of copious amounts of activated charcoal, or the intake of an appropriate antidote depending on the nature of the drug ingested. Apart from these existing methods, use of porous polymeric network for sequestration of overdosed drug is also getting investigated. Polymers and liposomes in the form of nanoparticles and nanocapsules have been explored for drug sequestration (Morey et al. 2004; Chakraborty \& Somasundaran, 2006; Dhanikula et al. 2007).

Among various types of sequestrants, subsized nano and micro polymeric particles, owing to their porous structure and large surface area will facilitate intense interaction between the polymer and the drug, allowing the drug to get sequestered in the porous polymeric network.

In recent years chitosan, an abundant natural polysaccharideobtained from crustacean exoskeletons has been extensively studied for drug delivery applications in the form of micro and nanoparticles, (Sinha et al. 2004, Hu et al. 2007; Papadimitriou et al. 2008; Bernkop-Schnürch \& Dünnhaupt 2012; Tian et al. 2016; Kong et al. 2017). Chitosan has been studied for tissue engineering (Hua et al. 2008, Bhardwaj et al. 2011; Sivashankari et al. 2016) and wound healing (Bae et al. 2009; Bano et al. 2017) applications. The benefits of chitosan based dietary foods has also been reported (Muzzarelli 1996; Li et al. 2013)

Since chitosan has been extensively studied for various applications where chitosan is ingested or implanted and proven to be benign, it can be 
explored for effective extraction of drugs from bulk media and to serve as scavengers for overdosed drugs. The reported work aimed at synthesis of subsized chitosan particles by a novel approach and to investigate their potential towards the sequestration of drugs. The subsized particles were synthesized inside the reverse micelles of benign surfactants Span 80 and Tween 80 . The prototype drug of choice was propafenone. Propafenone is an anti-arrhythmic drug, used to treat atrial and ventricular fibrillation. An acute overdose of this drug can lead to severe cardiac toxicity within 30 minutes to two hours of ingestion. Hence there is a need to find a remedy for its overdosing effects. The structure of propafenone is shown in Figure 1. It is hypothesized that one of the significant interactions of propafenone with chitosan will be through formation of hydrogen bonding.

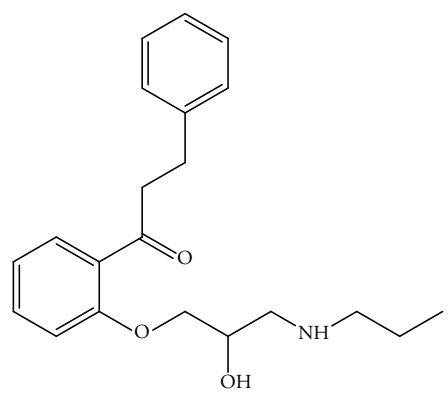

Figure 1. Chemical Structure of Propafenone, the AntiInflammatory Drug Used in the Present Study.

Apart from chitosan, Quat188 (glycidyl trimethylammonium chloride) modified chitosan has also been used for drug sequestration. Quat 188 is proven to enhance antimicrobial properties of chitosan(Kim et al. 2003). It also improves the interaction of chitosan with water due to the presence of quaternary ammonium ion. In this study Quat 188 was used to facilitate swelling of chitosan.

\section{EXPERIMENTAL}

Chemicals. Chitosan, sorbitan monooleate (Span80), Polyethylene glycol sorbitan monooleate (Tween80), toluene, acetone and Propafenone hydrochloride were purchased from Sigma-Aldrich. Glacial acetic acid was purchased from Fischer. Glutaraldehyde from Fluka and Quat 188 was obtained from Rohm and Haas Company.

Instruments. ${ }^{1} \mathrm{H}$ NMR Analysis. ${ }^{1} \mathrm{H}$ NMR spectrum was recorded at $25^{\circ} \mathrm{C}$ on a JEOL Lambda 400 $\mathrm{MHz}$ spectrometer. ${ }^{1} \mathrm{H}$ NMR chemical shifts in parts per million (ppm) were referenced relative to tetramethylsilane (TMS). $8.0 \mathrm{wt} \%$ of samples were dissolved in deuterated water $\left(\mathrm{D}_{2} \mathrm{O}\right)$. A trace amount of acetic acid was added to the sample to make chitosan soluble in $\mathrm{D}_{2} \mathrm{O}$.
SEM Analysis. The size range and morphology of the particles was determined using JSM-5310 Scanning microscope at $10 \mathrm{kV}$. The particles were placed on a copper wafer in vacuum and then sputter-coated with a gold layer before viewing.

UV Analysis. The amount of drug sequestered by the chitosan particles was quantified using UV-Vis analysis with a Shimadzu 2401 PC UV Spectrophotometer at $250 \mathrm{~nm}$.

Methodology. Modification of Chitosan with Glycidyl Trimethyl Ammonium Chloride (Quat 188). To prepare Quat 188-modified chitosan, 0.6g (3.75 × $10^{-3}$ mol per unit) chitosan was placed in a round bottom flask. To it $0.3525 \mathrm{~g}\left(1.88 \times 10^{-3} \mathrm{~mol}\right)$ of Quat 188 was added along with $10 \mathrm{~mL}$ of deionized water. The $\mathrm{pH}$ of the mixture was adjusted to $\mathrm{pH} 9$ by the dropwise addition of $1 \mathrm{M} \mathrm{NaOH}$ to convert Quat 188 to its epoxide form. The flask was sealed, purged with nitrogen and the reaction was performed at $70^{\circ}$ for 48 hours with continuous stirring. After the reaction, system was neutralized to $\mathrm{pH} 7$ and centrifuged to remove excess water. Quat 188-modified chitosan was further freeze dried using LABCONCO FREEZE DRY SYSTEM/ FREEZONE 45 freeze drier.

Fabrication of Microparticles of Chitosan and Quat188-modified Chitosan. The chitosan particles were fabricated inside the reverse micelle of Tween 80 and Span 80 . The chitosan solution was prepared by dissolving $2 \mathrm{~g}$ of chitosan in $200 \mathrm{~mL}$ of $2 \%(\mathrm{w} / \mathrm{w})$ acetic acid solution. $100 \mathrm{~mL}$ hexane, $2.6 \mathrm{~g}$ of Tween 80 and $3.4 \mathrm{~g}$ of Span 80 were placed in a round bottom flask. To it $2 \mathrm{~mL}$ of above chitosan solution was added drop wise under constant stirring. The system was allowed to stir for $1 \mathrm{~h}$ followed by dropwise addition of $0.1 \mathrm{~mL}$ glutaraldehyde stock solution (1.2 g glutaraldehyde/100 mL water). The system was further stirred for another $2 \mathrm{~h}$. Throughout this process the solution remained clear, and phase separation did not occur. After $2 \mathrm{~h}$ hexane was removed under reduced pressure by using a rotary evaporator. The product was washed several times with acetone to remove the surfactants. The particles were recovered by centrifugation. The particles were further freeze dried using LABCONCO FREEZE DRY SYSTEM/FREEZONE 45 freeze drier. A similar procedure was adopted to fabricate Quat188modified chitosan particles.

Sequestration Studies as a Function of Time, Initial Concentration, and NormalSaline. Drug sequestration studies were performed at $37^{\circ} \mathrm{C}$ in $\mathrm{pH} 7.4$ buffer to simulate the physiological condition of the human body. To study the sequestration of propafenone, a propafenone solution of certain concentration was 
added to $1 \mathrm{~mL}$ dispersion of chitosan particles $(2 \mathrm{mg} /$ $\mathrm{mL}$ of $\mathrm{pH} 7.4$ phosphate buffer). The system was equilibrated at $37^{\circ} \mathrm{C}$. The particles were separated from the drug solution system by filtration. The dispersion was filtered five times using a $0.2 \mu \mathrm{m}$ syringe filter to remove all the chitosan subparticles. The filtrate was subjected to UV-Vis analysis.

Sequestration experiments were done as a function of equilibration time and the initial free drug concentration. Experiments were carried out in triplicate to determine the standard deviation.

\section{RESULTS AND DISCUSSION}

Modification of Chitosan with (Quat 188) and Synthesis of Microparticles. A novel methodology was developed for the fabrication of modified and unmodified chitosan particles. Though various methods have been reported for the fabrication of chitosan micro and nanparticles (Liu et al. 2007; Wu et al. 2005; Dini et al. 2003), synthesis of chitosan microparticles by reverse microemulsion technique has not been reported yet.

Though attempts were made to disperse the droplets of chitosan solutions in nonsolvents such as toluene, olive oil, hexane etc. followed by crosslinking, micron size chitosan particles were not obtained by these methods.

Figure 2 shows the ${ }^{1} \mathrm{H}$ NMR spectrum of Quat 188modified chitosan in $\mathrm{D}_{2} \mathrm{O} .{ }^{1} \mathrm{H}$ NMR in $\mathrm{D}_{2} \mathrm{O}$ (in ppm): 4.3-4.25 [a: CH-O- of chitosan], 2.7-2.85 [b: $\mathrm{CH}-\mathrm{NH}_{2}$ of chitosan], 3.6-4.25 [c: CH-OH; d: $-\mathrm{O}-\mathrm{CH}$; e: $\mathrm{CH}-\mathrm{CH}_{2} \mathrm{OH}$; f: $\mathrm{CH}_{2} \mathrm{OH}$ of chitosan], 3.4-3.5 [1:- $\mathrm{NH}-\mathrm{CH}_{2-}$ of Quat 188], 4.3-4.25 [2: $\mathrm{CH}-\mathrm{OH}$ of Quat 188], 3.55-3.6 [3: $\mathrm{CH}_{2}-\mathrm{N}^{+}\left(\mathrm{CH}_{3}\right)$ of Quat 188], 3.0-3.3 [4: $\left(\mathrm{CH}_{3}\right)_{3}-\mathrm{N}^{+}$of Quat 188].

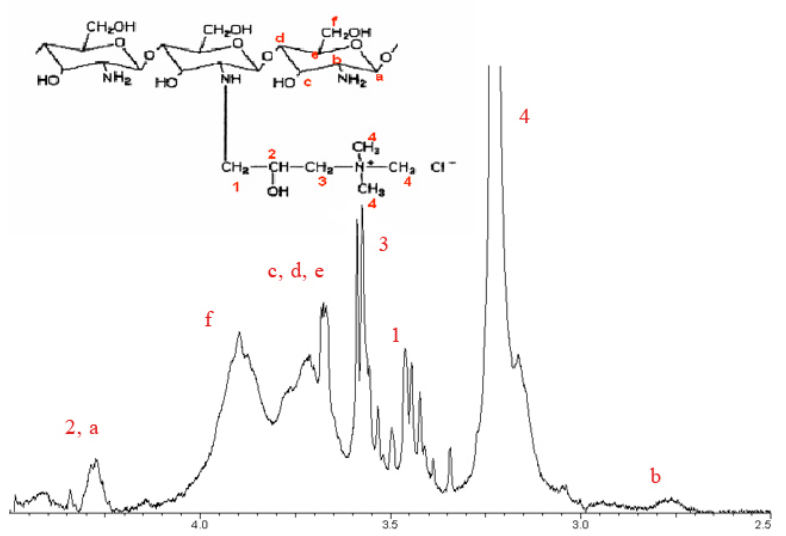

Figure 2. ${ }^{1} H$ NMR Spectrum of Quat 188-modified Chitosan.
SEM images as shown in Figures 3 reveals that the both chitosan and Quat 188-modified Chitosan particles were spherical, uniform in size with the average size of $10 \mu \mathrm{m}$.

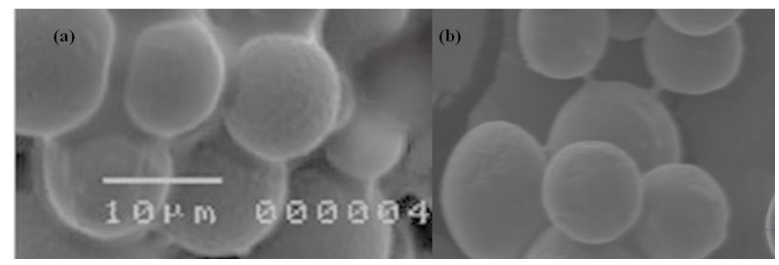

Figure 3. Scanning Electron Micrograph of a) Chitosan b) Quat 188-modified Chitosan Microparticles.

\section{Swelling Behavior of the Particles.}

Since the particles are intended for overdose drug sequestration, swelling is desirable for the encapsulation of the sequestrant, however swelling to a very large extent might cause release of the sequestrant from the substrate and hence increase the concentration of the free drug. To monitor the extent of swelling, $0.01 \mathrm{~g}$ of each type of particles were placed in $5 \mathrm{~mL}$ of a $\mathrm{pH} 7.4$ phosphate buffer maintained at $37{ }^{\circ} \mathrm{C}$ for certain time period. The particles were then centrifuged, and subjected to SEM analysis after pat drying. As shown in Figures 4 and 5, the SEM images show that the surface of the particles from both native and Quat 188-modified chitosan deformed upon exposure to the buffer solution for $10 \mathrm{~min}$. The surface crinkled more as the immersion time was extended to $2 \mathrm{~h}$ and swelled to almost twice the original size. Since the particles softened and swelled in limitation, they remain conducive for the drug sequestration application.

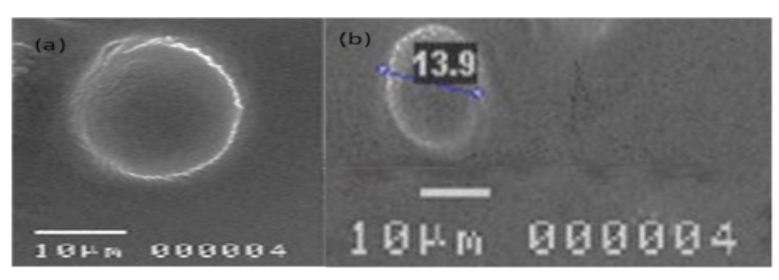

Figure 4. Scanning Electron Micrograph of a) Chitosan b) Quat 188-modified Chitosan Swelled for $10 \mathrm{~min}$.

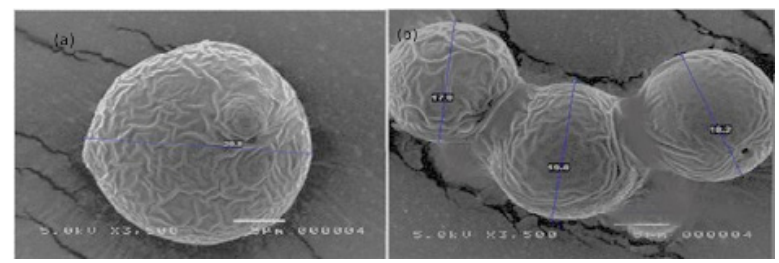

Figure 5. Scanning Electron Micrograph of a) Chitosan b) Quat 188-modified Chitosan Swelled for $2 \mathrm{~h}$.

Sequestration as a Function of Time. The sequestration of propafenone by chitosan and Quat 188-modified chitosan can be attributed to the interaction between propafenone and chitosan through hydrogen 
bonding and also to the diffusion of propafenone into the porous structure of the microparticles due to their swelling.

Drug sequestration experiments were performed at $37^{\circ} \mathrm{C}$ in $\mathrm{pH} 7.4$ buffer to simulate the physiological condition of the human body. Figure 6 shows the effect of equilibration time on drug sequestration by keeping the initial drug concentration constant.

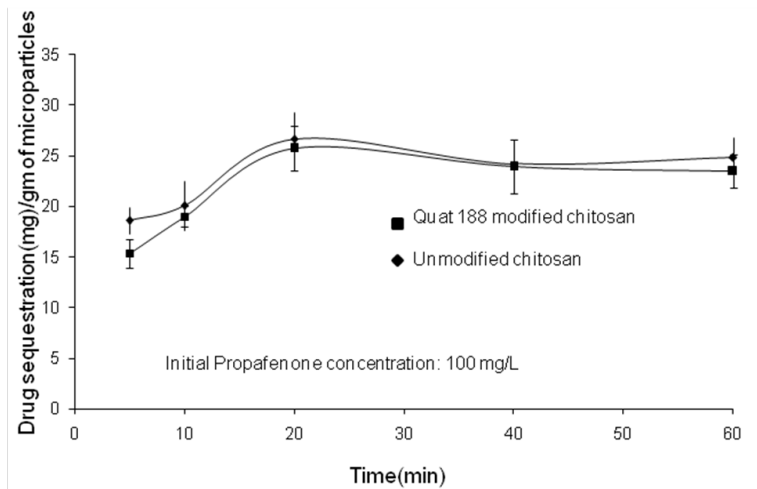

Figure 6. Effect of Equilibration Time on Propafenone Sequestration by Quat 188-modified and Unmodified Chitosan Microparticles.

It was observed that sequestration of drug by the chitosan microparticles increased as the equilibration time of the micro particles in contact with drug was increased from 5 to 20 minutes. In 5 min unmodified chitosan sequestered $19 \mathrm{mg}$ of propafenone, with time the amount sequestered enhanced to $27 \mathrm{mg}$ in 20 minutes which corresponds to $53 \%$ of the initial free drug. However, on further increase of equilibration time no improvement in the sequestration potential was observed. Particles might have swelled to the maximum extent within $20 \mathrm{~min}$ of immersion and hence facilitated maximum sequestration. Similar sequestration trend was observed for the Quat 188-modified chitosan microparticles. In $20 \mathrm{~min}$ Quat 188-modified chitosan sequestered $26 \mathrm{mg}$ of the initial free drug.

Sequestration Studies as a Function of Initial Concentration. The effect of the initial concentration variable on drug sequestration is seen in Figure 7. The trend reflects that increasing the free drug concentration from $50 \mathrm{mg} / \mathrm{L}$ to $200 \mathrm{mg} / \mathrm{L}$ increased the amount of drug sequestered by the particles in both modified and unmodified form. However, the amount of drug sequestered relative to the initial free drug concentration remained almost constant.

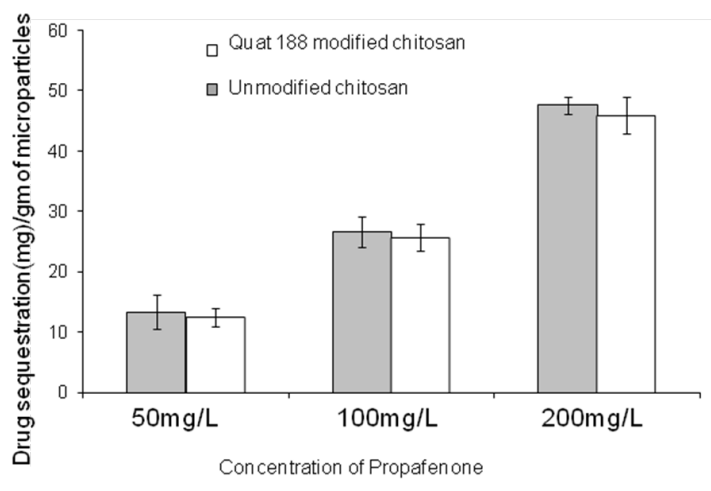

Figure 7. Effect of Initial Propafenone Concentration on Sequestration by Quat 188-Modified and Unmodified Chitosan Microparticles, Equilibration Time: $20 \mathrm{Min}$

Sequestration in the Presence of Normal Saline. Since human blood contains $0.9 \% \mathrm{w} / \mathrm{v} \mathrm{NaCl}$ (normal saline), the sequestration of propafenone in the presence of normal saline was investigated. In this case $0.9 \% \mathrm{w} / \mathrm{v} \mathrm{NaCl}$ was added to the buffer while performing the drug sequestration experiment as mentioned above. As seen in Fig 8, the addition of normal saline improved the binding interaction of the microparticles and propafenone.

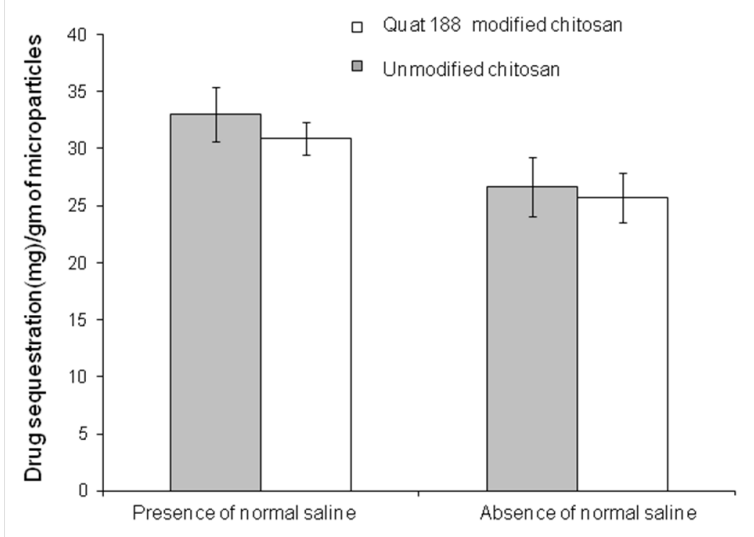

Figure 8. Sequestration of Propafenone in Normal Saline by Quat188-Modified and Unmodified Chitosan Particles, Initial Drug Concentration: 100 mg/mL, Equilibration Time: 20 Min.

Improved binding interaction can be attributed to enhanced swelling of the particles in the presence of normal saline. The cross-linked microspheres act as semi-permeable membranes. At $\mathrm{pH} 7.2$, the negatively charged acrylate ions drew free $\mathrm{Na}+$ ion inside the microspheres and induced a concentration imbalance in counterions between the inside of the microsphere and the surrounding solution. In order to minimize this chemical imbalance, water penetrated the microparticles resulting in swelling of the network. 
It can be observed that chitosan microparticles performed marginally better than Quat 188-modified chitosan particles towards propafenone sequestration. Quat 188-modification might have reduced the $\mathrm{H}$-bonding sites between propafenone and chitosan and reduced sequestration.

\section{CONCLUSIONS}

This study explored the feasibility of fabrication of chitosan microparticles in the reverse micelles of benign surfactants of Span 80 and Tween 80 and investigated the scope of utilizing them as drug sequestrant. The microparticles of Quat 188-modified chitosan and unmodified chitosan, fabricated by reverse micelle method were spherical and $10 \mu \mathrm{m}$ in diameter. The micron sized particles swelled and sequesterd propafenone. More than $50 \%$ of free drug was sequestered in 20 min both by modified and unmodified chitosan. Since the chitosan particles can bind to the free drug under physiological conditions, and the surfactants used for the synthesis is also benign, chitosan subparticles synthesized by this novel approach can be further studied for the sequestration drugs with similar structures such as serotonin, acetaminophen etc. which are intentionally consumed in excess in suicidal attempts.

\section{ACKNOWLEDGEMENT}

We are grateful to the Loyala Schools scholarly work faculty grant committee of Ateneo de Manila University for the financial support.

\section{REFERENCES}

Bae JW, Lee JH, Choi WS, Lee DS, Bae EH, Park KD. EPDIM peptide-immobilized porous chitosan beads for enhanced wound healing: Preparation, characterizations and in vitro evaluation. Mater Sci Eng C. 2009; 29(3):697-701.

Bano I, Arshad M, Yasin T, Afzal Ghauri M, Younus M. Chitosan: A potential biopolymer for wound management. Int J Biol Macromolec. 2017; 102: 380-383.

Bernkop-Schnürch A, Dünnhaupt S. Chitosanbased drug delivery systems. Eur J Pharm Biopharm. 2012; 81(3): 463-469.

Bhardwaj N, Nguyenqt, Chen AC, Kaplan DL, Sah RL. Potential of 3-D tissue constructs engineered from bovine chondrocytes/silk fibroin-chitosan for in vitro cartilage tissue engineering. Biomaterials. 2011; 32: 5773-5781.
Chakraborty S, Somasunadaran P. Sequestration of drugs using poly(acrylic acid) and alkyl modified poly(acrylic acid) nanoparticles. Soft Matter. 2006; 2:850-854.

Dhanikula A, Khalid N, Lee S, Yeung R, Risovic V, Wasan K, Leroux J. Long circulating lipid nanocapsules for drug detoxification. Biomaterials. 2007; 28: 1248-1257.

Dini J, Alexandridou S, Kiparissides C. Synthesis and characterization of cross-linked chitosan microspheres for drug delivery applications. J Microencapsulation. 2003; 20(3): 375-385.

Hu Y, Ding Y, Ding D, Sun M, Zhang L, Jiang $X$, Yang C. Hollow Chitosan/Poly(acrylic acid) Nanospheres as Drug Carriers. Biomacromolecules. 2007; 8:1069-1076.

Hua W, Ying W, Xiaoying C, Quan W. Proliferation of chondrocytes on porous poly(dl-lactide)/ chitosan scaffolds. Acta Biomaterialia. 2008; 4(1):76-87.

Kim JK, Lee JK, Lee TS, Park WH. Synthesis of chitooligosaccharide derivative with quaternary ammonium group and its antimicrobial activity against Streptococcus mutants. Int J Biol Macromol. 2003; 32: 23-27.

Kong M, Zuo Y, Wang M, Bai X, Feng C, Chen $\mathrm{X}$. Simply constructed chitosan nanocarriers with precise spatiotemporal control for efficient intracellular drug delivery. Carbohyd Polym. 2017; 169(1): 341-350.

Li J, Shi B, Yan S, Jin L, Guo Y. Li T, Guo X. Effects of dietary supplementation of chitosan on humoral and cellular immune function in weaned piglets. Anim Feed Sci Technol. 2013; 204-208.

Liu H, Fan H, Cui Y, Chen Y, Yao K, Goh JCH. Effects of the Controlled-Released Basic Fibroblast Growth Factor from Chitosan-Gelatin Microspheres on Human Fibroblasts Cultured on a ChitosanGelatin Scaffold. Biomacromolecules. 2007; 8:1446-1455.

Morey T, Varshney M, Flint J. Rajasekaran S, Shah D, Dennis D. Treatment of Local AnestheticInduced Cardiotoxicity Using Drug Scavenging Nanoparticles. Nano Letters. 2004; 4(4): 757-759.

Muzzarelli RAA. Chitosan-based dietary foods. Carbohyd Polym. 1996; 29(4): 309-316.

Papadimitriou S, Bikiaris D, Avgoustakis K, Karavas E, Georgarakis, M. Chitosan nanoparticles loaded 
with dorzolamide and pramipexole. Carbohydr Polym. 2008; 73: 44-54.

Sinha VR, Singla AK, Wadhawan S, Kaushik R, Kumria R, Bansal K, Dhawan S. Chitosan microspheres as a potential carrier for drugs. Int J Pharm. 2004; 274(1-2): 1-33.

Sivashankari PR, Prabaharan M. Prospects of chitosan-based scaffolds for growth factor release in tissue engineering. Int J Biol Macromolec. Part B, 2016; 93:1382-1389.

Tian Y, Sun Y, Wang X, Kasparis G, Mao S. Chitosan and its derivatives-based nano-formulations in drug delivery. Nanobiomaterials in Drug Delivery, 2016; 9: 515-572.

Wu Y, Yang W, Wang C, Hu J, Fu S. Chitosan nanoparticles as a novel delivery system for ammonium glycyrrhizinate, Int J Pharm. 2005; 295: 235-245. 